\title{
Position of the Academy of Nutrition and Dietetics, Dietitians of Canada, and the American College of Sports Medicine: Nutrition and Athletic Performance
}

\begin{abstract}
It is the position of the Academy of Nutrition and Dietetics, Dietitians of Canada, and the American College of Sports Medicine that the performance of, and recovery from, sporting activities are enhanced by wellchosen nutrition strategies. These organizations provide guidelines for the appropriate type, amount, and timing of intake of food, fluids, and supplements to promote optimal health and performance across different scenarios of training and competitive sport. This position paper was prepared for members of the Academy of Nutrition and Dietetics, Dietitians of Canada (DC), and American College of Sports Medicine (ACSM), other professional associations, government agencies, industry, and the public. It outlines the Academy's, DC's and ACSM's stance on nutrition factors that have been determined to influence athletic performance and emerging trends in the field of sports nutrition. Athletes should be referred to a registered dietitian/nutritionist for a personalized nutrition plan. In the United States and in Canada, the Certified Specialist in Sports Dietetics (CSSD) is a registered dietitian/ nutritionist and a credentialed sports nutrition expert.

(Can J Diet Pract Res. 2016;77:54)

(DOI: $10.3148 /$ cjdpr-2015-047)

Published at dcjournal.ca on 26 February 2016.
\end{abstract}

\begin{abstract}
RÉSUMÉ
L'Academy of Nutrition and Dietetics, Les diététistes du Canada et l'American College of Sports Medicine sont d'avis que des stratégies de nutrition soigneusement sélectionnées améliorent les performances sportives et la récupération à la suite d'activités physiques. Ces organismes proposent des lignes directrices quant au type et à la quantité d'aliments, de liquides et de suppléments à consommer et au moment approprié des apports pour favoriser une santé et une performance optimales dans divers scénarios d'entraînement et de sports de compétition. Cet énoncé de position a été préparé pour les membres de l'Academy of Nutrition and Dietetics, des Diététistes du Canada (DC) et de l'American College of Sports Medicine (ACSM), ainsi que pour d'autres associations professionnelles, des organismes gouvernementaux, l'industrie et le public. II décrit la position de l'Academy, des DC et de l'ACSM sur des facteurs propres à la nutrition qui influencent les performances athlétiques de même que les tendances émergentes dans le domaine de la nutrition du sport. Les athlètes devraient être orientés vers une ou un diététiste/nutritionniste pour obtenir un plan nutritionnel personnalisé. Aux États-Unis et au Canada, les Board Certified Specialists in Sports Dietetics (CSSD) sont à la fois des diététistes/ nutritionnistes et des experts en nutrition du sport qualifiés.

(Rev can prat rech diétét. 2016;77:54)

(DOI: 10.3148/cjdpr-2015-047)

Publié au dcjournal.ca le 26 février 2016.
\end{abstract}

\section{POSITION STATEMENT}

It is the position of the Academy of Nutrition and Dietetics, Dietitians of Canada, and the American College of Medicine that the performance of, and recovery from, sporting activities are enhanced by well-chosen nutrition strategies. These organizations provide guidelines for the appropriate type, amount and timing of intake of food, fluids and dietary supplements to promote optimal health and sport performance across different scenarios of training and competitive sport.

This paper outlines the current energy, nutrient, and fluid recommendations for active adults and competitive athletes. These general recommendations can be adjusted by sports dietitians to accommodate the unique issues of individual athletes regarding health, nutrient needs, performance goals, physique characteristics (i.e., body size, shape, growth, and composition), practical challenges and food preferences.

Access the Position Paper Nutrition and Athletic Performance at $w w w$.dietitians.ca/sports.

\section{ÉNONCÉ DE POSITION}

L'Academy of Nutrition and Dietetics, Les diététistes du Canada et l'American College of Sports Medicine sont d'avis que des stratégies de nutrition soigneusement sélectionnées améliorent les performances sportives et la récupération à la suite d'activités physiques. Ces organismes proposent des lignes directrices quant au type et à la quantité d'aliments, de liquides et de suppléments alimentaires à consommer et au moment approprié des apports pour favoriser une santé et une performance optimales dans divers scénarios d'entraînement et de sports de compétition.

Ce document décrit les recommandations actuelles en matière d'apport en énergie, en nutriments et en liquides pour les adultes actifs et les athlètes de compétition. Ces recommandations générales peuvent être ajustées par les diététistes du sport afin de convenir aux situations uniques des athlètes sur le plan de la santé, des besoins en nutriments, des objectifs de performance, des caractéristiques physiques (c.-à-d., taille, forme, croissance et composition du corps), des défis pratiques et des préférences alimentaires.

Accédez à l'énoncé de position sur la nutrition et la performance athlétique au www.dietetistes.ca/sport. 